\title{
Selecting Structural Innovations in DSGE models*
}

\author{
Filippo Ferroni \\ Chicago FED
}

\author{
Stefano Grassi \\ Department of Economics and Finance, \\ University of Rome 'Tor Vergata'
}

\author{
Miguel A. León-Ledesma \\ School of Economics and MaGHiC \\ University of Kent
}

March 8, 2018

\begin{abstract}
DSGE models are typically estimated assuming the existence of certain structural shocks that drive macroeconomic fluctuations. We analyze the consequences of estimating shocks that are "non-existent" and propose a method to select the economic shocks driving macroeconomic uncertainty. Forcing the these non-existing shocks in estimation produces a downward bias in the estimated internal persistence of the model. We show how these distortions can be reduced by using priors for standard deviations whose support includes zero. The method allows us to accurately select shocks and estimate model parameters with high precision. We revisit the empirical evidence on an industry standard medium-scale DSGE model and find that government and price markup shocks are innovations that do not generate statistically significant dynamics.
\end{abstract}

Keywords: Reduced rank covariance matrix, DSGE models, stochastic dimension search.

JEL Classification: C10, E27, E32.

*Ferroni: Chicago FED, 230 LaSalle, Chicago IL. Grassi: University of Rome 'Tor Vergata' and CREATES. León-Ledesma: School of Economics and Macroeconomics, Growth, and History Centre (MaGHiC), Keynes College, University of Kent, Canterbury, CT27NP, UK. An earlier version of this paper circulated with the title, 'Fundamental shock selection in DSGE models'. We wish to thank for helpful discussions Gianni Amisano, Benjamin Born, Fabio Canova, Samuel Hurtado, Alejandro Justiniano, Eric Leeper, Nels Lind, Christian Matthes, Luigi Paciello, Barbara Rossi, Juan Rubio-Ramirez, Luca Sala, Chris Sims, Paolo Surico, Leonardo Melosi, Andrea Tambalotti. The paper also benefited from comments of participants at the 2014 Working Group of Econometric Modeling at the ECB, the 2015 Annual Meeting of the Royal Economic Society, the 2015 Barcelona Summer Forum, the 2015 IAAE conference, and various seminars. Two anonymous referees contributed to improve the empirical part of the paper. Remaining errors are our own. The views in this paper are solely the responsibility of the authors and should not be interpreted as reflecting the views of the Federal Reserve Bank of Chicago or any other person associated with the Federal Reserve System. 


\section{INTRODUCTION}

One of the key challenges of modern macroeconomics rests on the identification of the sources of aggregate fluctuations. By specifying a coherent probabilistic structure of economically interpretable endogenous and exogenous processes, DSGE models represent ideal candidates to pin down the shocks driving business cycle fluctuations. ${ }^{1}$ A tacit but widespread assumption in the empirical literature on DSGE model estimation is that exogenous disturbances do exist in the sense that they capture aggregate economic uncertainty (up to a vector of idiosyncratic measurement errors). Common estimation practice implicitly "imposes" these structural shocks by restricting their standard deviation to be non-zero. In a Bayesian context, this assumption is reflected on the prior distributions imposed on the standard deviations of DSGE model shocks (e.g. typically an Inverse Gamma prior, IG henceforth). In classical statistics, standard deviations are re-parameterized by taking logarithmic transformations. In doing so, we rule out boundary solutions and, by construction, innovations always exist. ${ }^{2}$

In this paper, we address two related questions. First, we ask what are the consequences of imposing possibly non-existent shocks for the estimation of deep parameters of DSGE models. Second, we provide an easily implementable method to select the "true" shocks that drive macroeconomic fluctuations.

From an empirical point of view, there is mounting evidence that some of the structural DSGE shocks are unlikely to capture aggregate uncertainty and rather absorb misspecified propagation mechanisms of endogenous variables (see Schorfheide, 2013 for an overview). Moreover, it is not infrequent that shocks with dubious structural interpretation are used with the sole purpose of avoiding stochastic singularity and this complicates inference when they turn out to matter, say, for output or inflation fluctuations (see Chari et al., 2008 and Sala et al., 2010). This is an important question in modern stochastic models of economic fluctuations. Empirically, these models face two challenges. First, unveiling the structural innovations that set off fluctuations. Second, identifying the key transmission mechanisms that transform these innovations into business cycles. There is a large literature on the latter. However, because we impose the existence of a set of economic shocks, we do not yet understand what are the consequences for inference when estimating a vector of time series with an 'excessive' number of structural disturbances, i.e. what are the consequences for inference when estimating non-existent DSGE shocks? This is our first question mentioned above.

When taking the model to the data, we want to be able to test, rather than merely postulate, the existence of certain shocks. I.e., we want to be able to select which innovations are significant drivers of aggregate uncertainty. This is the second problem we tackle. To be able to estimate the possibly rank deficient covariance matrix of structural shocks, we need to 1) add idiosyncratic measurement errors

\footnotetext{
${ }^{1}$ See Smets and Wouters (2007) and Justiniano et al. (2010) amongst many others.

${ }^{2}$ We use the term "structural" shock throughout the paper to refer to shocks that have an economic interpretation and that affect the dynamics of the model. I.e. they are relevant sources of uncertainty. These shocks could also be termed "fundamental", but we prefer to term them structural to avoid confusion with the terminology used in other streams of the literature dealing with very different problems to ours.
} 
and 2) abandon standard IG priors and use distributions (univariate or multivariate) that allow for zero variances (or null eigenvalues).

Imposing a non-existent exogenous process has serious consequences for inference. In particular, we argue that it generates a downward bias in the estimate of the internal persistence of the model. In particular, when the econometrician assumes that the rank of the covariance matrix of economic shocks, $\Sigma$, is larger than the one of the true DGP, autoregressive parameters and parameters controlling the internal persistence of the model are grossly underestimated. Thus, we unveil a trade-off between including a wide set of potential sources of impulses (say in order to match more observable variables) and correctly identifying the model parameters that drive propagation. We then show that these distortions are reduced by considering priors on the structural shocks' covariance matrix that allow for rank deficiency. In the context of uncorrelated disturbances, truncated or un-truncated priors can be implemented as long as they attribute non-zero probability to zero standard deviations. Proper priors such as normal ${ }^{3}$ or exponential distributions have appealing properties since they allow us to recover existent and non-existent shocks in situations where the true number and combination of structural shocks is unknown ${ }^{4}$.

We explore the consequences of our approach in an empirical application and revisit the evidence of an industry-standard DSGE model. We estimate the Smets and Wouters (2007) model (SW hereafter) on seven key US quarterly macroeconomic time series, namely, the growth rate of real output, consumption, investment, wages, and hours worked, the inflation rate, and the short run interest rate. For comparability proposes, we consider the original data span, 1968-2004, and only depart from the baseline specification of the model by adding measurement errors on each observable and by assuming normal priors on the standard deviation of shocks. We identify as common sources of fluctuations technology shocks, monetary policy surprises, risk-premium, and investment demand shocks. Government spending and price markup shocks do not generate statistically significant transmission dynamics for the variables considered, only when assuming normal priors. Wage markup shocks are only marginally significant. The choice of the priors for standard deviations (STDs hereafter) matters for the estimates of the structural parameters of the SW model and, as a consequence, matters when studying the transmission mechanism of economic impulses (with non-zero variance). Relative to a model estimated without measurement errors, the responses of macro variables to risk-premium shocks are more persistent and hump shaped. When we impose the existence of structural and measurement error shocks (both with inverse gamma priors), we obtain unrealistically strong responses to monetary policy surprises. In this empirical application, we also find that prior specification comparisons in terms of marginal likelihoods are difficult to interpret as the

\footnotetext{
${ }^{3}$ Normal priors have support from $+\infty$ to $-\infty$, whereas standard deviations are positive. We discuss below how we exploit the uni- or bi-modality of the posterior to select which shocks are statistically non-existent.

${ }^{4}$ In the online appendix A.4 we consider a more general structural shocks covariance matrix where innovations can be correlated. We propose considering the conjugate Metropolis-within-Gibbs sampler proposed in Cúrdia and Reis (2010) adapted for rank deficient covariance matrices. In a rank deficient environment, we consider the singular inverse Wishart (IW) distribution (see Uhlig, 1994 and Díaz-García and Gutiérrez-Jáimez, 1997) and the conjugacy results in Díaz-García and Gutiérrez-Jáimez (2006).
} 
marginal likelihood estimates using inverse gamma priors are hugely influenced by the parameters shaping the prior (especially its location).

Our methodology is related to the literature on stochastic model specification search in state space models. We draw from Frühwirth-Schnatter and Wagner (2010) for the selection of structures in unobserved components models or in time varying parameter VAR models as in Belmonte et al. (2014) or Eisenstat et al. (2016). We build on that literature by proposing to estimate jointly the structural parameters and the stochastic specification of the DSGE shock structure. Our paper is also, albeit indirectly, related to the vast literature studying misspecification problems in DSGE model estimation. Invalid crossequation restrictions (e.g. Ireland, 2004, Del Negro and Schorfheide, 2009, Inoue et al., 2014), parameter instability of various forms (e.g. Fernández-Villaverde and Rubio-Ramírez, 2008, Galvao et al., 2015, Canova et al., 2015), incorrect assumptions about shock dynamics (Cúrdia and Reis, 2010), low frequency movements mismatches (e.g. Gorodnichenko and Ng, 2010, Ferroni, 2011, Canova, 2014), etc., may all plague inference in DSGE models. However, the literature so far is silent on the issue of interest of this paper. We are concerned with redundant model-based shocks which can generate distorted estimates and corrupt inference when forced to exist. ${ }^{5}$

The remainder paper is organized as follows. Section 2 presents the econometric setup and estimation procedures. Section 3 presents the inference distortions caused by incorrect assumptions about the rank of $\Sigma$. Section 4 presents the main results of our empirical investigation. Section 5 draws few concluding remarks.

\section{PRIORS FOR THE DSGE SHOCKS SELECTION}

Consider a DSGE model with (deep) parameters of interest $\Theta$. The (control and state) variables of the model, denoted by $s_{t}$, are driven by structural shocks with innovations $\epsilon_{t}$. The model is characterized by a set of equations that define the steady state values $s^{*}$ and Euler equations that describe the transition dynamics. Linearizing around the steady state gives a system of expectational difference equations that can be solved to yield a solution in the form of difference equations. The linearized solution of a DSGE has the following representation:

$$
s_{t+1}=\mathrm{A}(\boldsymbol{\Theta}) s_{t}+\mathrm{B}(\boldsymbol{\Theta}) \epsilon_{t+1} \quad \epsilon_{t+1} \sim \mathrm{N}_{r}\left(0, \Sigma_{\epsilon}\right),
$$

where A, B are nonlinear functions of the structural parameters of the model $\Theta, \epsilon_{t}$ is a $n \times 1$ vector of the structural innovations, $s_{t}$ is the $n_{s} \times 1$ vector of endogenous and exogenous states and $\Sigma_{\epsilon}$ is a covariance matrix of dimension $n \times n$ whose rank is $r=\operatorname{rank}\left(\Sigma_{\epsilon}\right) \leq n$. We denote by $\mathrm{N}_{r}\left(0, \Sigma_{\epsilon}\right)$ the $n \times 1$ multivariate

\footnotetext{
${ }^{5}$ There is a large literature on shocks "fundamentalness", in particular in the VAR context, see Lippi and Reichlin (1994) and subsequent literature. The concern in that literature focuses on the non-invertibility of the MA representation of the DSGE model. When not invertible, with the available set of observables, it is impossible to recover all the fundamental DSGE shocks using linear projections, i.e. VAR. Although in the text we may refer occasionally to "fundamental" sources of macroeconomic uncertainty, we are clearly not relating to this stream of work.
} 
singular Normal distribution with rank $r$. If the eigenvalues of $\mathrm{A}(\boldsymbol{\Theta})$ are inside the unit circle, the latter structure can be mapped into a $\operatorname{VMA}(\infty)$ (see Komunjer and $\mathrm{Ng}, 2011$ ). The mapping from the model based variables to a $n_{y} \times 1$ vector $y_{t}$ of observed times series is accomplished through a measurement equation augmented with series specific i.i.d. shocks in order to avoid the possibly stochastically singular model, as follows

$$
y_{j, t}=\Phi_{j}(\mathrm{~L} ; \boldsymbol{\Theta}) \epsilon_{t}+e_{j, t}, \quad \epsilon_{t} \sim \mathrm{N}\left(0, \Sigma_{\epsilon}\right), \quad e_{t} \sim \mathrm{N}(0, \Omega), \quad j=1, \ldots, n_{y}
$$

where $\Phi_{j}$ correspond to the $j$ th raw of $\Phi(\mathrm{L} ; \boldsymbol{\Theta})$, the MA polynomial of the DSGE model observable counterparts. The structural shocks and the measurement shocks are separately identifiable since the former are common and the latter are series specific. Moreover and more importantly, the measurement errors being i.i.d., they cannot explain the cross- and auto-correlation structure of the data, which is entirely determined by the common component, i.e. the DSGE model shocks and their MA structure.

Equation (2) can be seen as an approximate dynamic factor model where the row vector $\Phi_{j}(\mathrm{~L} ; \boldsymbol{\Theta})$ represents the factor loadings and $\epsilon_{t}$ the common factors (orthogonal to each other). There is, however, an important difference. While in the factor model we are interested in the number of factors, in this setup we are interested also in the combination of underlying common shocks since they have economic interpretations. This can be accomplished by studying the null space of $\Sigma_{\epsilon}$. If we assume $\Sigma_{\epsilon}$ diagonal, it is sufficient to check that standard deviations are not zero. If $\Sigma_{\epsilon}$ is a symmetric positive definite matrix, the non-zero eigenvalues correspond to the structural shocks. ${ }^{6}$

Following the literature on Bayesian stochastic variable selection in state space models initiated by Frühwirth-Schnatter and Wagner $(2010)^{7}$, we rewrite equation (1) as follows:

$$
\tilde{s}_{t+1}=\mathrm{A}(\boldsymbol{\Theta}) \tilde{s}_{t}+\mathrm{B}(\boldsymbol{\Theta}) \Sigma^{1 / 2} \tilde{\epsilon}_{t+1} \quad \tilde{\epsilon}_{t+1} \sim \mathrm{N}\left(0, \mathrm{I}_{n}\right)
$$

where $\mathrm{N}\left(0, \mathrm{I}_{n}\right)$ is the multivariate normal distribution with unitary variance. While the standard deviation of $\tilde{\epsilon}_{t+1}$ is fixed and normalized to one in estimation, the diagonal elements of $\Sigma^{1 / 2}$ are estimated. We consider classes of prior distributions for the diagonal elements of $\Sigma^{1 / 2}$ such that the probability of zero is positive, i.e. for $j=1, \ldots, n_{\epsilon}$ we assume that $\mathrm{P}\left(\sigma_{j}=0\right)>0$. It is important to notice that structural standard deviations are not identified up to sign switch, e.g. $\epsilon_{i} \sim \mathrm{N}\left(0, \sigma_{i}^{2}\right)= \pm \sigma_{i} \tilde{\epsilon} \sim \mathrm{N}(0,1)$. In other words, the model in the equation with $\left(-\Sigma^{1 / 2}\right)\left(-\tilde{\epsilon}_{t+1}\right)$ is observationally equivalent to the same model with $\Sigma^{1 / 2} \tilde{\epsilon}_{t+1}$. As a consequence, the likelihood function is symmetric around zero along the $\sigma_{j}$ dimension and bimodal if the true $\sigma_{j}$ is larger than zero. One could also, as it is standard practice, normalize the

\footnotetext{
${ }^{6}$ An alternative way to select the number and combination of structural DSGE shocks is to compute the marginal likelihood of model specifications with different combinations of shocks. However, this can be time consuming because it requires estimating each of the possible models. In models with typically 7 or 8 postulated structural shocks, the combinations of models to compare is very large and marginal likelihood comparisons will not be a very useful tool for selection. Our argument is even more persuasive for non-linear models for which the computation of the marginal likelihood is very burdensome.

${ }^{7}$ In the online appendix (section A.2) we briefly summarize the key ideas of this method and its correspondence with DSGE shock selection.
} 
sign to a positive value and estimate the standard deviations over a non-negative support. ${ }^{8}$ We propose to use the following priors:

1. Exponential priors:

$$
\sigma_{j} \sim \operatorname{Exp}\left(\lambda_{j}\right)
$$

With Exp priors, we fix the sign to be non-negative (but allowing for zero) prior to estimation. In order to assess the existence of specific shocks, we rely on the confidence sets of the posterior distribution and the statistical distance from zero.

2. Normal priors:

$$
\sigma_{j} \sim \mathrm{N}\left(\mu_{j}, \tau_{j}^{2}\right)
$$

In principle, the likelihood can be computed for positive and negative values of the standard deviation. Since the likelihood value is the same if the standard deviation is positive or negative, the likelihood function profile is symmetric around zero. Therefore, if a shock exists, the likelihood should be bi-modal, i.e. decreasing when we approach zero from the left or from the right. If a shock does not exists, the likelihood is uni-modal around zero, e.g. decreasing as we move away from zero. Accordingly, one can define a normal prior covering the entire real line support in order to exploit this feature of the likelihood. ${ }^{9}$

One can be interested in situations where structural disturbances are correlated. In such a case, a singular inverse Wishart prior can be imposed on the variance covariance matrix of the shocks. However, being somehow a non-standard practice on DSGE estimation, we leave the description and the derivation of this specific setup to the online appendix (Section A.4).

\section{INFERENCE DISTORTIONS WITH NON EXISTENT DSGE SHOCKS}

We now tackle the question of whether the introduction of non-existent shocks affects the estimation of parameters governing the impulse transmissions in the model. The conclusions derived here are based on intuitive arguments and Montecarlo exercises. While in this section we convey the intuition using a simple model, we also quantified the distortions induced in behavioral parameters and their policy implications an industry-standard New Keynesian DSGE. The results are summarized below and the exercises can be consulted in the online appendix sections A.5 and A.6.

We start first by studying the likelihood of the simplest DSGE model, a plain vanilla Real Business Cycle (RBC) model with inelastic labor supply, full capital depreciation, and an autoregressive process of

\footnotetext{
${ }^{8}$ This formulation is a simplification of the state space form proposed in Frühwirth-Schnatter and Wagner (2010) (FS-W) that is derived in online appendix (section A.2). The FS-W formulation is slightly more involved since their main goal is to have a closed form expression for the marginal likelihood which is not our focus here. In the results that follow, we also used their formulations and find no difference in the estimation results.

${ }^{9}$ Standard Bayesian simulators such as the RW Metropolis-Hastings can be employed to recover the posterior distribution with an additional random sign switch of the shocks' standard deviations. In the online appendix (section A.2) we describe the procedure to introduce the sign switch in an otherwise standard MCMC routine.
} 
order one for total factor productivity (TFP) shocks. In this setting, an analytical solution can easily be derived. ${ }^{10}$ We obtain the following recursive representation:

$$
\begin{aligned}
y_{t} & =z_{t}+\alpha k_{t}+e_{t} \quad e_{t} \sim \mathrm{N}\left(0, \sigma_{e}^{2}\right), \\
z_{t+1} & =\rho z_{t}+\sigma \epsilon_{t+1} \quad \epsilon_{t+1} \sim \mathrm{N}(0,1), \\
k_{t+1} & =\alpha k_{t}+z_{t},
\end{aligned}
$$

where small case variables indicate the log deviation of the variables from the non stochastic steady state. In particular, $k_{t}$ is capital per capita, $z_{t}$ is TFP, $y_{t}$ is output per capita, and $\alpha$ is the capital share in production. We assume that we observe neither the technology process nor capital. We observe output up to a measurement error, $e_{t} \sim \mathrm{N}\left(0, \sigma_{e}^{2}\right)$.

We run a controlled experiment to measure the impact of different priors on the shock standard deviations of the DSGE model. We simulate artificial data from the RBC model by calibrating structural parameters values to standard values in the literature, i.e. $\alpha=0.33, \rho=0.95$. We generate data assuming that the technology shock standard deviation is zero and positive. We fix the variance of $\sigma_{e}$ to 0.08 , i.e. the mean of the range of values of the structural standard deviation. The results obtained in this section are largely invariant to the values of structural and non-structural parameters used to generate data, to the the sample size, and to the location of priors and scale parameters. ${ }^{11}$

We generate 500 data points from the RBC model with the measurement error for each value of $\sigma$, and retain the last 100 for inference. We compute and estimate the posterior kernel of $\sigma$ assuming the following priors where $m$ stands for the mean, and $S D$ for the standard deviation:

- $\operatorname{IG}(\alpha, \beta)$ with $\alpha=2.0016$ and $\beta=0.2$ that gives $m=0.2$ and $S D=5$;

- $\operatorname{Normal}\left(\mu, \sigma^{2}\right)$ with $\mu=0.2$ and $\sigma^{2}=25$ that gives $m=0.2$ and $S D=5$;

- $\operatorname{Exp}(\lambda)$ with $\lambda=0.2$ that gives $m=5$ and $S D=5$.

With an abuse of notation we indicate parameter distribution (IG, N and Exp) and in parenthesis mean and standard deviations. While the measures of dispersion are the same across priors, the prior shape and support are different. It is important to notice that, while for the normal and exponential distribution third and higher moments exist, this is not true for the inverse gamma.

We first study the posterior kernel of $\sigma$ conditional on the other parameters being fixed at their true values. Being a unidimensional problem, we can directly plot the product of the likelihood times the prior (i.e. the posterior kernel) against different values of $\sigma$. This allows us to study the behavior of the kernel in the neighborhood of zero. Figure 1 displays the posterior kernel of $\sigma$ for a range of values of $\sigma$ $([-0.5,0.5])$ keeping the remaining parameters fixed at their true values. From the top left panel to the bottom right panel, we present the four cases for the values of the true standard deviation: $0,0.05,0.1$, 0.2. When the technology shock has zero variance, with a normal prior with loose precision the posterior

\footnotetext{
${ }^{10}$ See the online Appendix section A.1 for details.

${ }^{11}$ Results with different parameterization of the data generating process, scale and location parameters and sample size are available on request.
} 
Figure 1: Posterior kernels for different values of $\sigma$ 's. The figure reports the posteriors with: IG(0.2,5) prior (black dashed line); $N(0.2,5)$ prior (blue dotted line); Exp $(5,5)$ prior (red line). The values considered are $\sigma=\{0,0.05,0.10,0.20\}$.
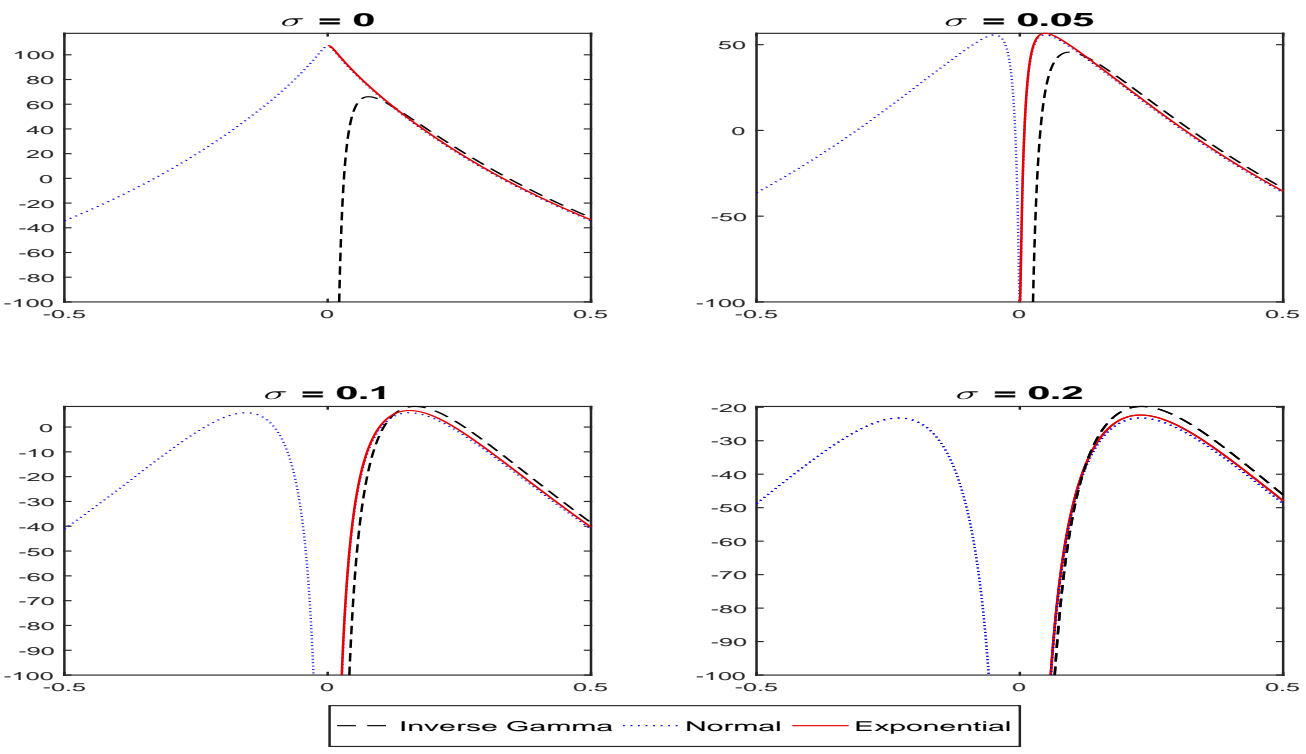

kernel of $\sigma$ is uni-modal centered on zero and with a tight standard deviation. Similar conclusions apply to the Exp prior, for which the posterior peaks at zero. Hence, the prior information on this parameter does not distort the information of the data likelihood. By assuming an IG prior, instead, we are forcing the kernel not to explore the region of the parameter space of a null variance and, as a consequence, we are corrupting the information contained in the data. Second, when the technology shock is non-zero the posterior kernel of $\sigma$ is similar across prior assumptions, and in the normal case the bi-modality of the likelihood is preserved. Therefore, normal or Exp priors do not seem to create distortions when the shock truly exists.

The posterior moments of the full set of parameters, $\alpha, \rho, \sigma$ and $\omega$, are computed using the Random Walk Metropolis-Hastings algorithm ${ }^{12}$ adapted for the sign switch when assuming normal priors for $\sigma$. We postulate a normal prior for $\alpha$ centered in 0.3 with 0.05 standard deviation, a beta distribution for $\rho$ centered in 0.5 with 0.2 standard deviation and an IG prior for the measurement error centered in 0.2 with a loose standard deviation of 4 .

Table 1 reports posterior statistics assuming different prior distributions and locations for the standard deviations. In particular, from top to bottom, for the inverse gamma prior and the normal, we move the prior location progressively away from zero. We do not report the the results for $\sigma>0$ since they are similar among priors and close to the true values; they can be consulted on the online appendix (Section B, Additional Tables and Graphs, Table 3).

There are three things to highlight. First, while the estimates of $\sigma$ are very imprecise with an IG prior,

\footnotetext{
${ }^{12}$ We launched two chains of 100,000 draws using as a starting point the mode of the posterior kernel.
} 
Table 1: Full MCMC estimates of model (3). The model is estimated using the Normal, the IG and the Exp prior for the standard deviations of structural shock. The table reports for all the structural parameters $\Theta$ : the posterior mode, the lower (5\%) and upper (95\%) quantile(credible set) and the marginal likelihood (ML) computed with the Laplace approximation and the modified armonic mean (in parenthesis).

\begin{tabular}{|c|c|c|c|}
\hline & \multicolumn{3}{|c|}{$\sigma=0$} \\
\hline \multirow[t]{2}{*}{$\Theta$} & Mode [Lower,Upper] & Mode [Lower,Upper] & Mode [Lower,Upper] \\
\hline & $\mathrm{IG}(\mathbf{0 . 0 5}, 5)$ & $\operatorname{Normal}(\mathbf{0 . 0 5}, 5)$ & $\operatorname{Exp}(\mathbf{5}, 5)$ \\
\hline$\alpha$ & $0.30[0.21,0.37$ & $0.30[0.22,0.38$ & $0.30[0.21,0.37$ \\
\hline$\rho$ & {$[0.10,0.61$} & $0.50[0.10,0.69]$ & $0.50[0.09,0.68]$ \\
\hline$\sigma$ & $0.01,0.04$ & $0.00[-0.05,0.04$ & $0.002[0.001,0.044]$ \\
\hline$\sigma_{e}$ & $0.09[0.08,0.11$ & $0.09[0.08,0.11]$ & $0.10[0.08,0.10]$ \\
\hline \multirow[t]{2}{*}{ ML } & $88.55(88.48)$ & $84.47(84.14)$ & $85.39(84.38)$ \\
\hline & $\operatorname{IG}(\mathbf{0 . 1}, 5)$ & $\operatorname{Normal}(\mathbf{0 . 1}, 5)$ & \\
\hline$\alpha$ & $0.29[0.21,0.37$ & $0.30[0.21,0.37$ & \\
\hline$\rho$ & $0.07,0.51$ & $0.50[0.10,0.69$ & \\
\hline$\sigma$ & $0.02,0.06$ & $0.00[-0.04,0.04$ & - \\
\hline$\sigma_{e}$ & {$[0.07,0.10$} & $0.09[0.08,0.11]$ & \\
\hline \multirow[t]{2}{*}{ ML } & $87.36(87.26)$ & $84.47(84.16)$ & \\
\hline & $\mathrm{IG}(\mathbf{0 . 2}, 5)$ & $\operatorname{Normal}(\mathbf{0 . 2}, 5)$ & \\
\hline$\alpha$ & $0.29[0.20,0.36$ & $0.29[0.21,0.38$ & \\
\hline$\rho$ & $0.04,0.40$ & $0.50[0.10,0.69$ & \\
\hline$\sigma$ & $0.06[0.04,0.08$ & $0.00[-0.05,0.04$ & - \\
\hline$\sigma_{e}$ & $0.08[0.06,0.10$ & $0.09[0.08,0.11]$ & \\
\hline ML & $84.58(84.49)$ & $84.47(84.16)$ & \\
\hline
\end{tabular}


the Normal and Exp priors with a sufficiently loose standard deviation allow the MCMC to explore more extensively the parameter space and hence to verify ex-post if the structural disturbance exists.

Second, while invariant for the normal prior, the marginal likelihood estimate depends on the location of the inverse gamma prior. The closer the hyper-parameter controlling the prior mean to zero, the larger the marginal likelihood. Hence, in principle, the marginal likelihood with inverse gamma distribution can be informative about the size of the shock. However, the marginal likelihood with inverse gamma priors is larger than the one obtained in the normal prior setup, which indeed is able to select the true DGP. This feature occurs systematically and it is not the artifact of one specific realization. ${ }^{13}$ While we do not have an analytical explanation for this result, intuitively, it may be related to the third and higher order moments of the probability distribution. For the normal distribution, all moments higher than the first are controlled by the scale parameter (squared variance) only. In the inverse gamma distribution, all moments depend on the shape and scale and, therefore, when changing the prior mean (keeping the dispersion fixed) we are implicitly changing the higher moments of the distribution (if they exist). This makes the comparison across distributions difficult to interpret and care should be taken when expressing a preference for the inverse gamma prior over the normal distribution by means of marginal likelihood. ${ }^{14}$ We will discuss this issue more extensively in the empirical section 4 below.

Third, when the true DGP has $\sigma=0$ and we impose an inverse gamma prior on $\sigma$, we obtain that the autoregressive parameter estimates are heavily downward biased. In the case of a null standard deviation, the posterior kernel displays a clear trade off between setting the standard deviation close to zero or reducing the persistence of the model dynamics. Since with IG priors we rule out null standard deviations, the posterior kernel of standard deviations does not include zero and, as a consequence, the structural shock has a dynamic impact on $y_{t}$. The only way to tone down the dynamic impact of this shock is to force the autoregressive parameter close to zero. To see this, assume that $\alpha=0$ and $\sigma_{e}^{2}=1$. Assume that the true DGP is the one with a null standard deviation, i.e. $\sigma=0$. We have that $y_{t}=e_{t}$ and the likelihood collapses to $\log \mathcal{L}\left(y_{T} \mid y^{T-1} ; \rho, \sigma=0\right) \propto-1 / 2 \sum_{t=1}^{T} y_{t}^{2}$. While $\rho$ is not identifiable in the true model, the persistence parameter becomes informative in the misspecified model. Suppose we work with a misspecified model in which $\sigma$ is fixed to a positive value, say $\delta>0$, which measures the degree of misspecification, i.e. the larger this value the more severe is the misspecification. The likelihood is given

\footnotetext{
${ }^{13}$ In the online appendix (section A.5), we report the marginal likelihood estimates based on 50 different datasets generated with $\sigma=0$ assuming either normal or inverse gamma distributions with mean 0.05 and standard deviation 5 . On average, there is a 4 to 5 log marginal likelihood difference in favor of the inverse gamma prior over the normal prior. This difference does not vanish with larger samples.

${ }^{14}$ In the online appendix (Section b, Additional Tables and Graphs, Table 6) we provide a decomposition of the (log-) marginal likelihood into a constant, the determinant of the inverse Hessian, the prior, and the likelihood for different locations of the priors. The differences in the marginal likelihood when using inverse gamma or normal priors are due entirely to the prior and not to the likelihood or the determinant of the inverse Hessian.
} 
by:

$$
\begin{gathered}
\log \mathcal{L}\left(y_{T} \mid y^{T-1} ; \rho, \sigma=\delta\right) \propto-1 / 2 \sum_{t=1}^{T} \log \left(s_{t}+1\right)-1 / 2 \sum_{t=1}^{T} \frac{\left(y_{t}-z_{t \mid t-1}\right)^{2}}{1+s_{t}} \\
z_{t+1 \mid t}=\left(1-k_{t}\right) z_{t \mid t-1}+k_{t} y_{t}, \quad s_{t+1}=k_{t}+\delta^{2}, \quad k_{t}=\rho \frac{s_{t}}{1+s_{t}},
\end{gathered}
$$

where the recursions are derived from the Kalman filter with $s_{1}=\delta^{2} /\left(1-\rho^{2}\right)$ and $z_{1 \mid 0}=0 .{ }^{15}$ In order to minimize the information discrepancy between the misspecified model likelihood, i.e. $\mathcal{L}\left(y_{T} \mid y^{T-1} ; \rho, \sigma=\delta\right)$, and the true DGP model likelihood, i.e. $\mathcal{L}\left(y_{T} \mid y^{T-1} ; \rho, \sigma=0\right)$, the autoregressive parameter has to go to zero. When $\rho=0$, we have $k_{t}=0, z_{t \mid t-1}=0, s_{t}=\delta^{2}$, and the likelihood becomes:

$$
\log \mathcal{L}\left(y_{T} \mid y^{T-1} ; \rho=0, \sigma=\delta\right) \propto-T / 2 \log \left(\delta^{2}+1\right)-\sum_{t=1}^{T} 1 / 2 \frac{y_{t}^{2}}{1+\delta^{2}} .
$$

which minimizes the distance to the likelihood of the true DGP.

\subsection{Further Discussion}

In fully fledged DSGE models, the persistence of model dynamics is controlled not only by the autoregressive parameters, but also by the deep parameters capturing real and nominal frictions in the economy. In a battery of controlled Montecarlo exercises, we found that when shutting down some economic shocks in the DSGE model as presented in Smets and Wouters (2007) (henceforth SW) a substantial downward bias in the parameters estimates arise when using IG priors, e.g. the parameters controlling price and wage rigidity. Similarly, the dynamic transmission of shocks and variance decomposition are substantially distorted when forcing all the shocks to exist with inverse gamma priors. Since we do not know ex-ante what are the key shocks driving aggregate fluctuations, IG priors are problematic as they may induce biases in estimated parameters that can be sizable. Normal or Exp priors do not suffer from any particular disadvantage when confronted with data that are generated by an unknown number of structural disturbances. We tested these priors on different values of the standard deviations of the structural shocks, of the measurement errors (including zeros) and with various sample sizes. The results of these exercises can be consulted in the online appendix.

It is also important to highlight that the non-existence results for some of the innovations are not to be interpreted as 'hard zeros'. While the posterior mode of the standard deviation of the some shock can be zero, the posterior distribution of this parameter will have some probability mass on positive values. Notice also that the autoregressive parameters of non existing shocks are not identifiable. However, since we do not know ex-ante what the non-existing shocks are, we also ignore for which exogenous processes can we identify the persistence coefficient. As a consequence, when estimating the model with the full set of shocks, the likelihood along the dimension of the autoregressive parameter of these non-existing processes is flat and the posterior overlaps with the prior. While this does not cause any problem per se, it makes

\footnotetext{
${ }^{15}$ See Durbin and Koopman (2012) for an introduction.
} 
the estimation inefficient. To cope with this, applied researchers can run a two step estimation procedure. In the first stage, they estimate the full set of exogenous processes' parameters (i.e. standard deviations and autoregressive parameters) and identify the structural sources of fluctuations. In the second stage, they can fix the autoregressive parameters of the non-existing shocks obtained in the first stage to avoid the non-identifiability of the persistence coefficient.

A crucial assumption of our approach is that the vector of observed times series is generated by a combination of structural and measurement shocks. In the absence of measurement errors, the DSGE model with a rank deficient covariance matrix is stochastically singular and, as a consequence, impossible to estimate with likelihood based approaches. The inclusion of measurement error allows us to avoid the stochastic singularity problem. One may argue that measurement error sweeps the rest of the variability of observables that is not explained by economic shocks. However, since structural shocks are common factors and measurement errors are variable-specific shocks, when measurement errors capture a larger proportion of the variability of a particular observable, it is precisely indicating that some postulated structural shocks may not be true common factors.

\section{Structural inNOVATIONS AND THE ROLE OF PRIORS FOR STANDARD DEVIATIONS}

We now study the role played by priors on structural DSGE shocks by reconsidering the estimates of a standard DSGE model using US macroeconomic data. We keep as benchmark the SW model. ${ }^{16}$ Although there are many more applications of interest, here we focus on the question of whether some of the standard impulses assumed in the existing literature are true drivers of macroeconomic fluctuations and not just the artifact of avoiding stochastic singularity. Moreover, we try to assess the empirical relevance of the choice of priors for STD for the transmission of DSGE shocks.

The SW model is estimated based on seven quarterly macroeconomic time series. Output growth is measured as the percentage growth rate of Real GDP, consumption growth as the percentage growth rate of personal consumption expenditure deflated by the GDP deflator, and investment growth as the percentage growth rate of Fixed Private Domestic Investment deflated by the GDP deflator. Hourly compensation is divided by the GDP price deflator in order to obtain the real wage variable. The aggregate real variables are expressed in per capita terms using population over 16. Inflation is the first difference of the log of the Implicit Price Deflator of GDP, and the interest rate is the Federal Funds Rate divided by four. For comparability of estimates, we consider the same data span as in SW, 1968-2004. The mapping between

\footnotetext{
${ }^{16}$ In the appendix, we provide the log-linearized equations and a brief description of the parameters estimated and calibrates.
} 
observables and model based quantities is accomplished through the following measurement equations

$$
\begin{aligned}
\text { output growth } & =\bar{\gamma}+\Delta y_{t}+\omega_{y} e_{y, t}, \\
\text { consumption growth } & =\bar{\gamma}+\Delta c_{t}+\omega_{c} e_{c, t}, \\
\text { investment growth } & =\bar{\gamma}+\Delta i_{t}+\omega_{i} e_{i, t}, \\
\text { real wage growth } & =\bar{\gamma}+\Delta w_{t}+\omega_{w} e_{w, t}, \\
\text { hours } & =\bar{l}+l_{t}+\omega_{l} e_{l, t}, \\
\text { inflation } & =\bar{\pi}+\pi_{t}+\omega_{p} e_{p, t}, \\
f f r & =\bar{\beta}+R_{t}+\omega_{r} e_{r, t}, \\
e_{x, t} & \sim \mathrm{N}(0,1) \text { with } x=y, c, i, w, l, p, r,
\end{aligned}
$$

where all variables are measured in percent. $\bar{\pi}$ and $\bar{\beta}$ measure the steady state level of net inflation and short term nominal interest rates, respectively, $\bar{\gamma}$ captures the deterministic long-run growth rate of real variables, and $\bar{l}$ captures the mean of hours. $e_{x, t}$ are standardized normal i.i.d. measurement error (ME) shocks.

We estimate and fix the same parameters as in SW with one exception. Relative to the original SW specification, we assume that the impact of technology on government spending, $\rho_{g a}$ in their model, is zero so that the government spending process is independent from the technology process. Priors for the structural parameters but STD are the same as in SW. We estimate three main specifications (and several variants, see next section):

1. The first specification $(I G)$ coincides with the original SW setup. In this specification, we assume that measurement error shocks are zero, i.e. $\omega_{x}=0$ for $x=y, c, i, w, l, p, r$, and structural shock standard deviations have an IG prior with mean 0.1 and standard deviation of 2 .

2. In the second specification, we assume an $I G$ prior on both measurement errors and structural shocks with a very loose standard deviation (e.g. 10). We consider two variants that differ in terms of prior location:

(a) One variant $(I G I G)$, a 'conventional' one, where the mean for the structural shocks is centered in 0.1 and for the measurement error in 0.2 .

(b) One variant $\left(I G I G_{*}\right)$ where the prior for measurement error is centered in 0.4 and for structural shocks in 0.2 . The latter is chosen based on marginal likelihood considerations, i.e. by selecting the hyper-parameters on a discrete grid of values that maximize the marginal data density.

3. In the third specification, $(N N)$, we postulate that the standard deviations of structural shocks and measurement errors are normally distributed ${ }^{17}$ with a very loose standard deviation of 10 and with

\footnotetext{
${ }^{17}$ Given that the results using Exp and normal priors in the previous section were very similar, we report here the results using normal priors only.
} 
location $0.1 .^{18}$ So, in principle, we allow for the possibility that some structural and/or measurement shocks are zero.

Table 2 reports the posterior mean and $90 \%$ confidence bands for a selection of structural parameters and the STD of shocks.

Table 2: Posterior median and $90 \%$ confidence bands of a selection of structural parameters in the four specifications: IG, $I G I G_{*}, I G I G$ and $N N$. Bottom part, Laplace approximation with decomposition.

\begin{tabular}{|c|c|c|c|c|c|c|c|c|}
\hline & & $N N$ & & $I G I G_{*}$ & & $I G I G$ & & $I G$ \\
\hline$\iota_{p}$ & 0.46 & $0.23,0.71]$ & 0.49 & $0.26,0.72]$ & 0.46 & $0.22,0.69]$ & 0.30 & $0.13,0.48]$ \\
\hline$\phi$ & 2.85 & $2.00,4.02]$ & 2.23 & $1.18,3.50]$ & 2.24 & $1.14,3.57]$ & 6.07 & $4.35,7.84]$ \\
\hline$\xi_{w}$ & 0.79 & $0.70,0.87]$ & 0.78 & $0.68,0.86]$ & 0.78 & $0.69,0.87]$ & 0.69 & $0.57,0.80]$ \\
\hline$\lambda$ & 0.76 & $0.64,0.86]$ & 0.69 & $0.55,0.83]$ & 0.73 & $0.60,0.86]$ & 0.69 & $0.62,0.76]$ \\
\hline$r_{y}$ & 0.15 & $0.09,0.20]$ & 0.16 & $0.11,0.22]$ & 0.16 & $0.10,0.21]$ & 0.09 & $0.05,0.12]$ \\
\hline$\rho_{b}$ & 0.69 & $0.55,0.82]$ & 0.77 & $0.65,0.87]$ & 0.77 & $0.65,0.87]$ & 0.23 & $0.09,0.38]$ \\
\hline$\rho_{r}$ & 0.19 & $0.04,0.37]$ & 0.55 & $0.21,0.91]$ & 0.34 & $0.10,0.90]$ & 0.16 & $0.05,0.27]$ \\
\hline$\sigma_{a}$ & 0.36 & $0.31,0.41]$ & 0.34 & $0.28,0.39]$ & 0.34 & $0.30,0.39]$ & 0.44 & $0.40,0.49]$ \\
\hline$\sigma_{b}$ & 0.11 & $0.08,0.14]$ & 0.10 & $0.07,0.13]$ & 0.09 & $0.07,0.12]$ & 0.24 & $0.20,0.28]$ \\
\hline$\sigma_{g}$ & -0.01 & $-0.21,0.22]$ & 0.13 & $0.07,0.22]$ & 0.08 & $0.04,0.18]$ & 0.58 & $0.53,0.63]$ \\
\hline$\sigma_{i}$ & 0.22 & $0.11,0.34]$ & 0.17 & $0.08,0.31]$ & 0.11 & $0.04,0.29]$ & 0.40 & $0.33,0.49]$ \\
\hline$\sigma_{r}$ & 0.21 & $0.11,0.25]$ & 0.13 & $0.07,0.19]$ & 0.15 & $0.06,0.22]$ & 0.24 & $0.22,0.27]$ \\
\hline$\sigma_{p}$ & -0.04 & $-0.17,0.16]$ & 0.10 & $0.06,0.15]$ & 0.08 & $0.03,0.16]$ & 0.13 & $0.10,0.15]$ \\
\hline$\sigma_{w}$ & 0.03 & $0.01,0.10]$ & 0.08 & $0.05,0.13]$ & 0.05 & $0.03,0.09]$ & 0.26 & $0.22,0.30]$ \\
\hline log Laplace Approximation (A) & & -893.2 & & -848.8 & & -852.3 & & -904.6 \\
\hline $\log$ Constant & & 38.6 & & 38.6 & & 38.6 & & 32.2 \\
\hline log det Hessian & & -117.1 & & -123.1 & & -124.1 & & -110.5 \\
\hline $\log$ Prior $(\mathrm{B})$ & & -47.2 & & 4.9 & & 4.4 & & -25.1 \\
\hline log Likelihood & & -767.5 & & -769.2 & & -771.3 & & -801.1 \\
\hline$(\mathrm{A})-(\mathrm{B})$ & & -846.0 & & -853.7 & & -856.8 & & -879.4 \\
\hline
\end{tabular}

There are a number of relevant results to highlight. First, government spending $(g)$ shocks and price markup $(p)$ shocks are estimated to be 'non-existent', since the posterior support for their standard deviations includes zero and their posterior distributions are unimodal (not shown here). Technology $(a)$, investment $(i)$, risk-premium $(b)$ or 'liquidity' shocks ${ }^{19}$, and monetary policy shocks $(r)$ are instead estimated to be structural. The wage markup shock, however, is only marginally so. ${ }^{20}$ For the $I G I G$ specifications, the estimated STD of government spending and price markup shocks are smaller than in the case with no measurement error $(I G)$ yet still significantly different from zero. ${ }^{21}$

\footnotetext{
${ }^{18}$ The location parameter for the Normal prior is not very influential. See next section.

${ }^{19}$ As shown in Fisher (2015), the SW risk premium or preference shock can be interpreted as a structural shock to the demand for safe and liquid assets such as short-term Treasury securities.

${ }^{20}$ If the model is estimated for rolling sub-samples, the shock appears not to be structural for the majority of the sub-samples. Results available on request.

${ }^{21}$ It is important to note that the fact that, for instance, shocks to government spending are found not to be structural in the
} 
Second, the posterior moments of deep parameters appear to be estimated differently in the three setups. For the estimates of $\phi$, the second derivative of the investment adjustment cost function, the mean estimate of $N N$ is significantly smaller than the value in $I G(6.07)$ and larger than the values obtained with the IGIG specifications. This figure is closer to the value (2.48) available in Christiano et al. $(2005)^{22}$; by using a IRF matching they do not need to specify and estimate the whole shock structure of the SW model. Moreover, the price indexation parameter, $\iota_{p}$, is 0.30 for the $I G$ model and the $N N$ estimated parameter is substantially larger. Albeit to a smaller degree, similar conclusions apply for the estimate of $\xi_{w}$ and $\lambda$, the wage stickiness and habit parameter respectively. Overall, estimates of deep parameters change when we introduce measurement error shocks and allow for the possibility of zero STD. Structural parameter estimates of the specifications where we assume IG on both measurement error and structural shocks are similar to the $N N$ ones, with one important exception. The estimated persistence of the monetary policy shock in the two IGIG specifications are different from the $N N$ (and the $I G)$ specification. From an economic standpoint, this parameter captures the extent to which current monetary policy surprises are predictable using past shocks. We find that the persistence of the monetary policy surprises is estimated to be 0.55 in the $I G I G_{*}$ model and 0.34 in the $I G I G$ model as opposed to values below 0.2 in the $N N$ or $I G$ cases. Besides the fact that the former values have weaker economic interpretation, this parameter is crucial for the dynamic transmission of monetary policy shocks as we will review in the next section.

Third, the table compares the marginal likelihood (ML) achieved by the different specifications by means of Laplace Approximations. The latter is then broken down into its main components: the log prior, the log likelihood (the sum of the one step ahead forecast errors in the Kalman Filter), the log of the determinant of the Hessian (measuring the curvature of the likelihood around the mode), and the constant (proportional to the sample size and to the number of parameters). The table shows that there are large differences in ML and the highest values are attained with $I G I G$ specifications. However, this result is entirely driven by the prior. With the $I G I G$ or the $I G I G^{*}$ specification, we neither describe better the observed data nor we have more curvature around the mode. If we remove the prior contribution from the ML, the resultant would rank the $N N$ specification first. As pointed out in section 3, we believe that comparing marginal likelihoods across specifications with IG and N priors is misleading; as much as using it as a device for model selection.

In sum, normal priors identify as common sources of fluctuations technology shocks, monetary policy surprises, risk-premium/liquidity shocks, investment demand shocks, and possibly wage markup shocks. Accordingly, many structural parameters estimates change. This is due partly to the introduction of

$N N$ specification does not imply that government spending is fixed. What this means is that these shocks, within the context of the SW model, are not significant drivers of macroeconomic uncertainty. This could be the case if, for instance, these changes to $g$ were fully expected by agents.

${ }^{22}$ Quoting Christiano et al. (2005), "[1/ $\left.\phi\right]$ is the elasticity of investment with respect to a 1 percent temporary increase in the current price of installed capital. Our point estimate implies that this elasticity is equal to 0.40 ". 
measurement errors and partly to the prior assumptions about the origin of fluctuations. We investigate this further below.

\subsection{The Dynamic transmission of the STRUCTURAL SOURCES OF FLUCTUATIONS}

To gauge the extent to which the assumptions about the existence of shocks affect the transmission dynamics of the model, we study the impulse response functions of four variables of interest to the identified sources of fluctuations. We thus neglect the response to government and price markup shocks as they do not generate significant transmission dynamics in the $N N$ specification (while they do with the $I G$ ones).

We focus our attention on the response of output growth, inflation, interest rate, and hours worked to a supply shock (technology), to a demand shock (risk-premium/liquidity shock), and to a monetary policy surprise. Figure 2 reports the mean estimated impulse response functions under different specifications. The benchmark SW case $(I G)$ is reported with black triangles, the $N N$ specification with blue dots (with $90 \%$ gray confidence bands) and the $I G I G_{*}$ and $I G I G$ specifications in red circles and green diamonds respectively. We have normalized the impact of the shocks across specifications. In particular, a monetary policy impulse generates the same impact increase in the interest rate, a supply impulse the same impact increase in output and a demand impulse the same impact increase in consumption (not shown here). We do not report the response function to demand and supply shocks for the $I G I G$ specifications because they are very similar to the $N N$ one.

There are two important remarks arising from this picture. First, the dynamic transmissions of demand and supply shocks are statistically different between the original SW setup $(I G)$ and the specification where we are agnostic about the origins of fluctuations $(N N)$. While supply shocks generate a negative co-movement between hours and output in both settings, the magnitude is smaller and less persistent in the $N N$ case (see the first row). After a technology shock, in the original SW setup, hours worked need 12 quarters to revert back to steady state, while less then 5 in the $N N$ setup. Demand shocks (second row) generate impulse response functions that are hump-shaped and more persistent with normal priors, with a peak delay of $2 / 3$ quarters. ${ }^{23}$

Second, the prior assumptions about shock standard deviations matter for the estimated transmission dynamics of the model. The third row of figure 2 reports the transmission mechanism of monetary policy surprises under the four different settings. In the original SW setup and the specification where we are agnostic about the sources of fluctuations $(N N)$, an unexpected increase in the federal fund rate of 15 basis points generates a decline in inflation of less then 5 basis points at the peak of the effect and a decline in output of less then 20 basis points. These figures are very close to those obtained in empirical studies using VARs (see Ramey, 2016).

\footnotetext{
${ }^{23}$ Differences in propagation and in the existence or non-existence of shocks give rise to different model conclusions. E.g. in the online appendix we report the historical shock decomposition of output growth in terms of different economic shocks with the $I G$ and the $N N$ setting. The differences are stark.
} 
Figure 2: Transmission mechanism of structural shocks. From top to bottom, dynamic transmission of supply (technology), demand (liquidity preference) and monetary policy shocks to output, hours, inflation and interest rate (left to right). Blue dots NN median estimates and gray areas the $90 \%$ confidence sets. Black triangles represent the estimates with $I G$, green diamonds IGIG and red circles IGIG* .
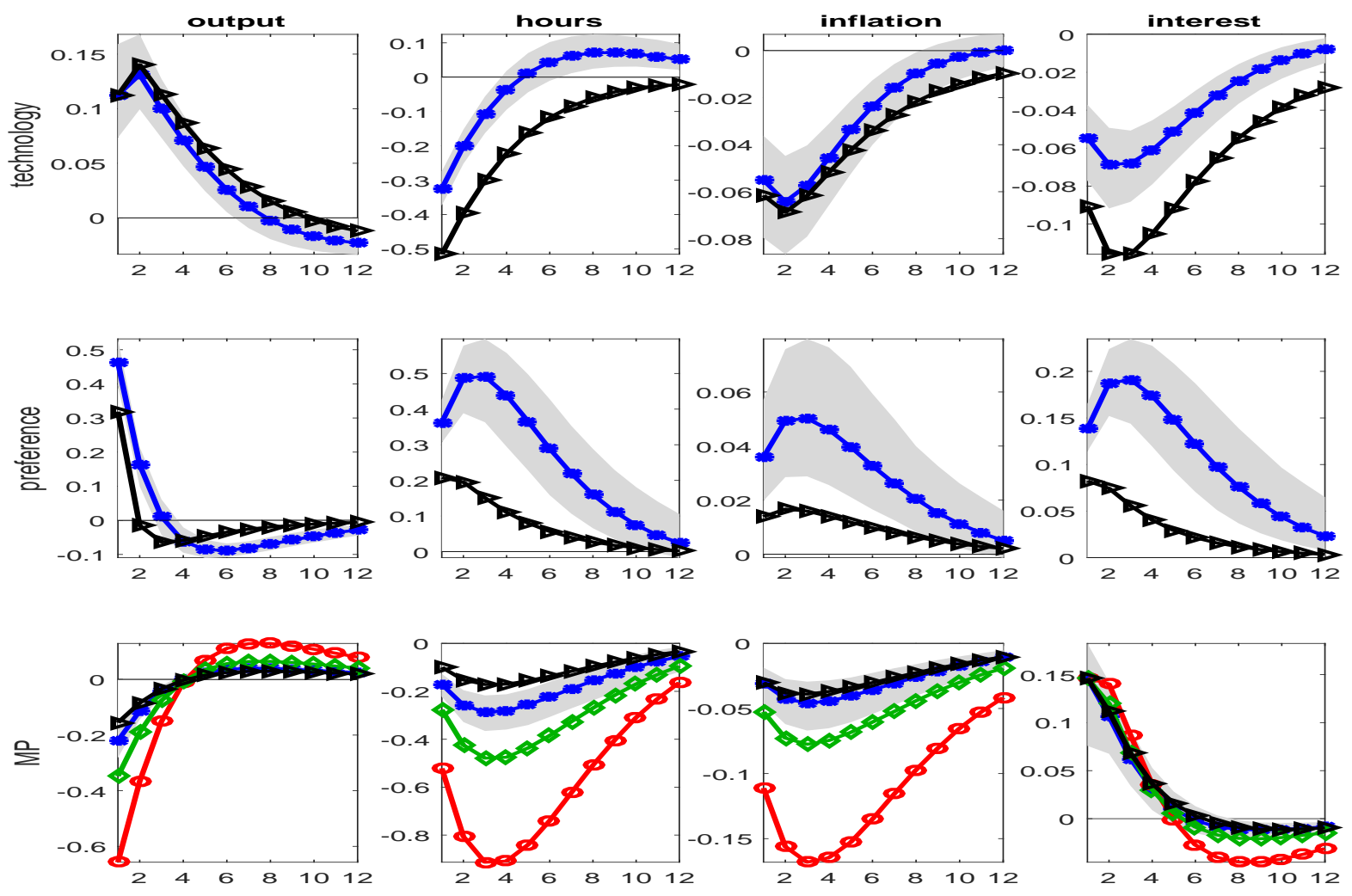

Bands $N N \rightarrow N N \multimap I G I G * \bullet I G I G \rightarrow I G$

In the setup with inverse gamma priors on structural and measurement errors, the same increase generates a huge response in inflation and output, between twice to more than three times larger. ${ }^{24}$ Why does this difference arise? In the $I G I G$ settings we are forcing all the shocks to exist, both structural and measurement errors. When this happens, the 'residuals' in the interest rate measurement equation are forced to be explained by both a monetary policy shocks and by the interest rate measurement errors. With a small (0.16) $\rho_{r}$ monetary policy shocks and measurement error are difficult to separately identify as they are both close to be i.i.d. So whenever the likelihood moves toward regions where the measurement error STD is large, it prefers to assign different persistence to these two shocks in order to be able to distinguish them. Since it cannot do this for the measurement error, it does it for the monetary policy shock. Hence the large $\rho_{r}$, which generates a statistically large and economically implausible transmission mechanism.

\footnotetext{
${ }^{24}$ It is also noticeable the large difference between $I G I G$ and $I G I G_{*}$, which indicates that the choice of prior location matters for these models. This is investigated further in section 4.2 below.
} 
Unfortunately, being highly misspecified, this class of models are typically subject to measurement errors, and large measurement error shocks tend to be associated with high values of the marginal likelihood. Hence, when choosing the $I G I G$ specification with the highest marginal likelihood $\left(I G I G_{*}\right)$, a researcher would be lead even more astray from a plausible representation of a MP shock.

In all, the choice of the priors for STD matters in practice. When identifying the economic sources of fluctuations and when studying the transmission mechanism of structural shocks. It is in this sense that we uncover a trade-off between imposing a wide set of potential sources of uncertainty and the estimation of the parameters that drive propagation.

\subsection{The SENSITIVITy OF THE POSTERIOR TO PRIORS ON THE STANDARD DEVIATION}

When we study models with more shocks than observables (e.g. as the SW model with measurement errors), the likelihood has something to say about the most likely combination of shocks, i.e. the model specification. As a consequence, the STD prior distribution does matter for the posterior analysis. Depending on the location and dispersion, the prior density could favor or penalize the likelihood of a specific model shock configuration. As a result, it might influence posterior analysis; ideally, one would like to work with priors whose hyper-parameters matter little for posterior analysis. If this is not the case, one should be aware of the extent to which the priors on STDs influence posterior analysis. ${ }^{25}$

Postulating a normal prior on STD is largely uninfluential for the computation of the posterior parameter distributions and of the marginal likelihood (as long as we have a sufficiently loose precision). It does not really matter if we postulate, a priori, that the STD of the measurement error on, say, interest rate is close to zero or not. The $N N$ setting generates estimates of the posterior distributions and of the marginal data density that are invariant to the prior mean.

This appealing property of the normal prior on shocks' STD does not carry over for the $I G$ distribution, where the posterior parameter and the marginal likelihood estimates are very sensitive to the location parameter. In such a case, different configurations of prior means lead to different estimates of the parameters and of the marginal likelihood; and arguably to different transmission mechanisms of the innovations. We found large differences in parameters estimates and marginal likelihoods, of as much as $20 \log$ points, even for prior locations that are relatively close. However, the marginal likelihood comparisons can be confusing as they might be driven by the priors, as it is case in Table 4.

Normal priors overcome all these concerns. They offer a natural benchmark for selecting the fundamental drivers of economic fluctuations and they are insensitive to the choice of prior hyper-parameters.

\footnotetext{
${ }^{25}$ In the online appendix, we document the sensitivity of the posterior estimates of parameters and marginal likelihood when using inverse gamma priors on shocks' standard deviations in the context of the SW model with measurement errors. In this section, we summarized the key results of this analysis.
} 


\section{Conclusions}

One of the key questions in macroeconomics concerns the identification of the structural impulses that set off macroeconomic fluctuations, the other key question being the identification of the propagation mechanisms that transform shocks into business cycles. Estimated DSGE models have become the standard methodology to address this question as they provide a coherent and economically interpretable structure. However, the widespread assumption when estimating DSGE models with likelihood methods is that certain exogenous shocks do exist in the sense that they capture macroeconomic uncertainty. We offer reasons for questioning this assumption and for adopting priors that allow us to test, rather than assume, the existence of economic shocks.

We show that postulating the existence of a non-existing exogenous processes generates a substantial downward bias in the estimates of the parameters driving internal persistence of the model. Thus, we unveil a tradeoff between the inclusion of a potentially large number of structural innovations and estimates of the parameters driving propagation. To prevent this problem, we propose an easily implementable strategy of using normal (or exponential) priors on the standard deviation of shocks together with measurement errors to avoid stochastic singularity. Our simulation evidence shows that these priors allow us to select the true structural shocks entering the DSGE model and that the remaining parameters are estimated with precision.

We analyzed the evidence on the existence of structural shocks in the medium-scale New Keynesian model of Smets and Wouters (2007). We find that government spending and price markup shocks are innovations that do not generate statistically significant dynamics, with the wage markup shock being only marginally significant. Hence, they are not fundamental sources of macroeconomic fluctuations in the context of that model. Technology, investment, risk-premium, and monetary policy surprises are all found to be important for the sample and the set of observables considered. By allowing or forcing all these (and measurement errors) shocks to play a role, substantial differences emerge in terms of the estimated parameters and of transmission mechanism of economic shocks.

\section{REFERENCES}

Belmonte, M. A. G., Koop, G., and Korobilis, D. (2014). Hierarchical Shrinkage in Time-Varying Parameter Models. Journal of Forecasting, 33:80-94.

Canova, F. (2014). Bridging DSGE Model and Raw Data. Journal of Monetary Economics, 57:132-145.

Canova, F., Ferroni, F., and Matthes, C. (2015). Approximating Time Varying Structural Model with Time Invariant Structures. CEPR Discussion Papers N. 10803.

Chari, V. V., Kehoe, P. J., and McGrattan, E. R. (2008). New Keynesian Models: Not Yet Useful for Policy Analysis. American Economic Journal: Macroeconomics, 1:24-66. 
Christiano, L. J., Eichenbaum, M., and Evans, C. L. (2005). Nominal Rigidities and the Dynamic Effects of a Shock to Monetary Policy. Journal of Political Economy, 113:1-45.

Cúrdia, V. and Reis, R. (2010). Correlated Disturbances and U.S. Business Cycles. NBER Working Papers 15774, National Bureau of Economic Research, Inc.

Del Negro, M. and Schorfheide, F. (2009). Monetary Policy Analysis with Potentially Misspecified Models. American Economic Review, 99:1415-1450.

Díaz-García, J. A. and Gutiérrez-Jáimez, R. (1997). Proof of the conjectures of H. Uhlig on the singular multivaraite beta and the Jacobian of a certain tranformation. Annals of Statistics, 25:2018-2033.

Díaz-García, J. A. and Gutiérrez-Jáimez, R. (2006). Distribution of the generalized inverse of a random matrix and its applications. Journal of Statistical Planning and Inference, 136:183-192.

Durbin, J. and Koopman, S. J. (2012). Time Series Analysis by State Stace Methods. Oxford University Press, second edition.

Eisenstat, E., Chan, J. C., and Strachan, R. W. (2016). Stochastic Model Specification Search for TimeVarying Parameter VARs. Econometrics Review, 35:1638-1665.

Fernández-Villaverde, J. and Rubio-Ramírez, J. F. (2008). How Structural Are Structural Parameters? In NBER Macroeconomics Annual 200\%, Volume 22, NBER Chapters, pages 83-137. National Bureau of Economic Research, Inc.

Ferroni, F. (2011). Trend Agnostic One-Step Estimation of DSGE Models. The B.E. Journal of Macroeconomics, 11:1-36.

Fisher, J. D. (2015). On the Structural Interpretation of the Smets-Wouters 'Risk Premium' Shock. Journal of Money, Credit and Banking, 47:511-516.

Frühwirth-Schnatter, S. and Wagner, H. (2010). Stochastic Model Specification Search for Gaussian and Partial non-Gaussian State Space Models. Journal of Econometrics, 154:85-100.

Galvao, A., Giraitis, L., Kapetanios, G., and Petrova, K. (2015). Local Bayesian Estimation and Forecasting with a TVP Model. Mimeo, Mimeo.

Gorodnichenko, Y. and Ng, S. (2010). Estimation of DSGE Models when the Data are Persistent. Journal of Monetary Economics, 57:325-340.

Inoue, A., Kuo, C.-H., and Rossi, B. (2014). Identifying the Sources of Model Misspecification. CEPR Discussion Papers 10140, C.E.P.R. Discussion Papers. 
Ireland, P. N. (2004). A Method for Taking Models to the Data. Journal of Economic Dynamics and Control, 28:1205-1226.

Justiniano, A., Primiceri, G. E., and Tambalotti, A. (2010). Investment Shocks and Business Cycles. Journal of Monetary Economics, 57:132-145.

Komunjer, I. and Ng, S. (2011). Dynamic Identification of Dynamic Stochastic General Equilibrium Models. Econometrica, 79:1995-2032.

Lippi, M. and Reichlin, L. (1994). VAR Analysis, Nonfundamental Representations, Blaschke Matrices. Journal of Econometrics, 63:307-325.

Ramey, V. A. (2016). Macroeconomic shocks and their propagation. Handbook of Macroeconomics, 2:71 $-162$.

Sala, L., Soderstrom, U., and Trigari, A. (2010). The Output Gap, the Labor Wedge, and the Dynamic Behavior of Hours. Working Paper Series 246, Sveriges Riksbank (Central Bank of Sweden).

Schorfheide, F. (2013). Estimation and Evaluation of DSGE Models: Progress and Challenges. In Advances in Economics and Econometrics: Theory and Applications, Tenth World Congress.

Smets, F. and Wouters, R. (2007). Shocks and Frictions in US Business Cycles: A Bayesian DSGE Approach. American Economic Review, 97:586-606.

Uhlig, H. (1994). On Singular Wishart and Singular Multivariate Beta Distributions. Annals of Statistics, 22:395-405. 


\section{A Appendix}

\section{A.1 Smets And Wouters (2007) MOdel}

The log linearized equilibrium conditions are summarized as follows:

$$
\begin{aligned}
y_{t} & =c / y c_{t}+i / y i_{t}+r^{k} k / y z_{t}+e_{t}^{g} \\
c_{t} & =c_{1} c_{t-1}+\left(1-c_{1}\right) E c_{t+1}+c_{2}\left(h_{t}-E h_{t+1}\right)-c_{3}\left(r_{t}-E \pi_{t+1}+e_{t}^{b}\right) \\
i_{t} & =i_{1} i_{t-1}+\left(1-i_{1}\right) E_{t} i_{t+1}+i_{2} q_{t}+\epsilon_{t}^{i} \\
q_{t} & =q_{1} E q_{t+1}+\left(1-q_{1}\right) E r_{t+1}^{k}-\left(r_{t}-E \pi_{t+1}+e_{t}^{b}\right) \\
y_{t} & =\alpha \phi_{p} k_{t}+(1-\alpha) \phi_{p} h_{t}+\phi_{p} \epsilon_{t}^{a} \\
k_{t}^{s} & =k_{t-1}+z_{t} \\
z_{t} & =\psi /(1-\psi) r_{t}^{k} \\
k_{t} & =k_{1} k_{t-1}+\left(1-k_{1}\right) i_{t}+k_{2} \epsilon_{t}^{i} \\
m p_{t} & =\alpha\left(k_{t}^{s}-h_{t}\right)+e_{t}^{a}-w_{t} \\
\pi_{t} & =\pi_{1} \pi_{t-1}+\pi_{2} E \pi_{t+1}-\pi_{3} m p_{t}+e_{t}^{p} \\
r_{t}^{k} & =-\left(k_{t}-h_{t}\right)+w_{t} \\
m w_{t} & =w_{t}-\left(\sigma_{n} h_{t}+\frac{1}{1+\lambda / \gamma}\left(c_{t}-\lambda / \gamma c_{t-1}\right)\right) \\
w_{t} & =w_{1} w_{t-1}+\left(1-w_{1}\right) E\left(\pi_{t+1}+w_{t+1}\right)-w_{2} \pi_{t}+w_{3} \pi_{t-1}+m w_{t}+e_{t}^{w} \\
R_{t} & =\rho_{R} R_{t-1}+\left(1-\rho_{R}\right)\left(\rho_{\pi} \pi_{t}+\rho_{y}\left(y_{t}-y_{t}^{f}\right)+\rho_{\Delta y} \Delta\left(y_{t}-y_{t}^{f}\right)\right)+e_{t}^{r} \\
& + \text { flexible economy equations }
\end{aligned}
$$

where variables with time subscript are variables from the original non linear model expressed in log deviation from the steady state. Flexible output is defined as the level of output that would prevail under flexible prices and wages in the absence of the two mark-up shocks. Seven structural shocks. The model has five AR(1), government, technology, preference, investment specific, monetary policy, and two ARMA $(1,1)$ processes, price and wage markup.

$$
\begin{aligned}
c_{1} & =\lambda / \gamma(1+\lambda / \gamma), \quad c_{2}=\left[\left(\sigma_{c}-1\right)\left(W^{h} h / C\right)\right] /\left[\sigma_{c}(1+\lambda / \gamma)\right], \quad c_{3}=(1-\lambda / \gamma) /(1+\lambda / \gamma) \sigma_{c}, \quad k_{1}=(1-\delta) / \gamma \\
k_{2} & =(1-(1-\delta) / \gamma)\left(1+\beta \gamma^{1-\sigma_{c}}\right) \gamma^{2} \phi, \quad i_{1}=1 /\left(1+\beta \gamma^{1 \sigma_{c}}\right), \quad i_{2}=\left(1 /\left(1+\beta \gamma^{1-\sigma_{c}}\right) \gamma^{2} \phi \quad q_{1}=\beta \gamma^{-\sigma_{c}}(1-\delta),\right. \\
\pi_{1} & =i_{p} /\left(1+\beta \gamma^{1-\sigma_{c}} i_{p}\right), \quad \pi_{2}=\beta \gamma^{1-\sigma_{c}} /\left(1+\beta \gamma^{1-\sigma_{c}} i_{p}\right), \\
\pi_{3} & \left.\left.=1 /\left(1+\beta \gamma^{1-\sigma_{c}} i_{p}\right)\left[\left(1-\beta \gamma^{1-\sigma_{c}} \xi_{p}\right)\left(1-\xi_{p}\right) / \xi_{p}\left(1+\left(\phi_{p}-1\right) \epsilon_{p}\right)\right)\right)\right], \quad w_{1}=1 /\left(1+\beta \gamma^{1-\sigma_{c}}\right), \\
w_{2} & =\left(1+\beta \gamma^{1-\sigma_{c}} i_{w}\right) /\left(1+\beta \gamma^{1-\sigma_{c}}\right), \quad w_{3}=i_{w} /\left(1+\beta \gamma^{1-\sigma_{c}}\right), \\
w_{4} & =1 /\left(1+\beta \gamma^{1-\sigma_{c}}\right)\left[\left(1-\beta \gamma^{1-\sigma_{c}} \xi_{w}\right)\left(1-\xi_{w}\right) / \xi_{w}\left(1+\left(\phi_{w}-1\right) \epsilon_{w}\right)\right], \\
\bar{\gamma} & =100(\gamma-1), \quad \bar{\pi}=100\left(\pi_{*}-1\right), \quad \bar{\beta}=\left(\left(\pi_{*} /\left(\beta * \gamma^{\sigma_{c}}\right)\right)-1\right) * 100
\end{aligned}
$$

The coefficients are function of the deep parameters of the model which are summarized and described in table 3 . 
Table 3: The table reports the parameter notation (first column), the parameter description (second column) and posterior mean estimated using MCMC or the parameters that are fixed (third column).

\begin{tabular}{|c|c|c|}
\hline$\Theta$ & Description & SW mean or fixed values \\
\hline$\gamma$ & slope of the deterministic trend in technology & 1.004 \\
\hline$\delta$ & depreciation rate & 0.025 \\
\hline$\varepsilon_{p}$ & good markets kimball aggregator & 10 \\
\hline$\varepsilon_{w}$ & labor markets kimball aggregator & 10 \\
\hline$\lambda_{w}$ & elasticity of substitution labor & 1.5 \\
\hline$c g$ & gov't consumption output share & 0.18 \\
\hline$\beta$ & time discount factor & 0.998 \\
\hline$\phi_{p}$ & 1 plus the share of fixed cost in production & 1.61 \\
\hline$\phi$ & inverse of the elasticity of investment relative to installed capital & 5.74 \\
\hline$\alpha$ & capital share & 0.19 \\
\hline$\lambda$ & habit in consumption & 0.71 \\
\hline$\xi_{w}$ & wage stickiness & 0.73 \\
\hline$\xi_{p}$ & price stickiness & 0.65 \\
\hline$i_{w}$ & wage indexation & 0.59 \\
\hline$i_{p}$ & price indexation & 0.47 \\
\hline$\sigma_{n}$ & elasticity of labor supply & 1.92 \\
\hline$\sigma_{c}$ & intertemporal elasticity of substitution & 1.39 \\
\hline$\psi$ & st. st. elasticity of capital adjustment costs & 0.54 \\
\hline$\rho_{\pi}$ & monetary policy response to $\pi$ & 2.04 \\
\hline$\rho_{R}$ & monetary policy autoregressive coeff. & 0.81 \\
\hline$\rho_{y}$ & monetary policy response to y & 0.08 \\
\hline$\rho_{\Delta y}$ & monetary policy response to y growth & 0.22 \\
\hline$\rho_{a}$ & technology autoregressive coeff. & 0.95 \\
\hline$\rho_{g}$ & gov spending autoregressive coeff. & 0.97 \\
\hline$\rho_{g a}$ & cross coefficient tech-gov & 0 \\
\hline$\rho_{b}$ & technology autoregressive coeff. & 0.21 \\
\hline$\rho_{q}$ & technology autoregressive coeff. & 0.71 \\
\hline$\rho_{m}$ & monetary policy autoregressive coeff. & 0.15 \\
\hline$\rho_{p}$ & price markup autoregressive coeff. & 0.89 \\
\hline$\rho_{w}$ & wage markup autoregressive coeff. & 0.96 \\
\hline$\mu_{w}$ & wage markup ma coeff. & 0 \\
\hline$\mu_{w}$ & wage markup ma coeff. & 0 \\
\hline$\sigma_{a}$ & sd technology & 0.45 \\
\hline$\sigma_{g}$ & sd government spending & 0.52 \\
\hline$\sigma_{b}$ & sd preference & 0.25 \\
\hline$\sigma_{r}$ & sd monetary policy & 0.24 \\
\hline$\sigma_{q}$ & sd investment & 0 \\
\hline$\sigma_{w}$ & sd wage markup & 0 \\
\hline$\sigma_{p}$ & sd price markup & 0 \\
\hline
\end{tabular}


Table 4: Posterior median and $90 \%$ confidence bands of the three specifications, IG, IGIG and NN under different prior locations.

\begin{tabular}{|c|c|c|c|c|c|c|c|c|}
\hline & & $N N$ & & $I G I G_{*}$ & & $I G I G$ & & $I G$ \\
\hline$\iota_{w}$ & 0.55 & $0.31,0.77]$ & 0.62 & {$[0.39,0.83]$} & 0.58 & {$[0.35,0.81]$} & 0.62 & {$[0.41,0.82]$} \\
\hline$\iota_{p}$ & 0.46 & $0.23,0.71]$ & 0.49 & $0.26,0.72]$ & 0.46 & $0.22,0.69]$ & 0.30 & $0.13,0.48]$ \\
\hline$\psi$ & 0.52 & $0.33,0.72]$ & 0.51 & $0.31,0.71]$ & 0.52 & $0.32,0.72]$ & 0.61 & $0.44,0.77]$ \\
\hline$\phi_{p}$ & 1.43 & $1.30,1.57]$ & 1.42 & $1.29,1.56]$ & 1.41 & $1.28,1.55]$ & 1.71 & $1.58,1.84]$ \\
\hline$\alpha$ & 0.22 & $0.18,0.27]$ & 0.23 & $0.18,0.27]$ & 0.23 & $0.19,0.27]$ & 0.20 & $0.16,0.23]$ \\
\hline$\phi$ & 2.85 & $2.00,4.02]$ & 2.23 & $1.18,3.50]$ & 2.24 & $1.14,3.57]$ & 6.07 & $4.35,7.84]$ \\
\hline$\sigma_{c}$ & 1.03 & $0.87,1.23]$ & 1.05 & $0.88,1.25]$ & 1.03 & $0.89,1.19]$ & 1.49 & $1.24,1.75]$ \\
\hline$\sigma_{l}$ & 1.48 & $0.38,2.50]$ & 1.46 & $0.33,2.48]$ & 1.35 & $0.25,2.30]$ & 1.75 & $0.84,2.71]$ \\
\hline$\xi_{w}$ & 0.79 & $0.70,0.87]$ & 0.78 & $0.68,0.86]$ & 0.78 & $0.69,0.87]$ & 0.69 & $0.57,0.80]$ \\
\hline$\xi_{p}$ & 0.61 & $0.52,0.69]$ & 0.60 & $0.52,0.68]$ & 0.61 & $0.52,0.69]$ & 0.67 & {$[0.58,0.75]$} \\
\hline$\lambda$ & 0.76 & $0.64,0.86]$ & 0.69 & $0.55,0.83]$ & 0.73 & $0.60,0.86]$ & 0.69 & $0.62,0.76]$ \\
\hline$r_{\pi}$ & 1.97 & $1.69,2.27]$ & 2.05 & $1.78,2.35]$ & 2.03 & $1.74,2.33]$ & 2.02 & $1.73,2.32]$ \\
\hline$r_{R}$ & 0.83 & $0.78,0.88]$ & 0.82 & $0.72,0.88]$ & 0.83 & $0.74,0.89]$ & 0.81 & $0.76,0.85]$ \\
\hline$r_{y}$ & 0.15 & $0.09,0.20]$ & 0.16 & $0.11,0.22]$ & 0.16 & $0.10,0.21]$ & 0.09 & $0.05,0.12]$ \\
\hline$r_{\Delta y}$ & 0.25 & $0.20,0.29]$ & 0.24 & $0.19,0.29]$ & 0.23 & $0.18,0.28]$ & 0.22 & $0.18,0.27$ \\
\hline $\bar{\gamma}$ & 0.45 & $0.40,0.49]$ & 0.44 & $0.40,0.49]$ & 0.45 & $0.40,0.49]$ & 0.43 & {$[0.40,0.45]$} \\
\hline $\bar{\pi}$ & 0.67 & $0.51,0.84]$ & 0.68 & 0.51, & 0.67 & 0.51, & 0.83 & $0.66,1.01]$ \\
\hline $\bar{\beta}$ & 0.22 & $0.11,0.34]$ & 0.22 & $0.10,0.34]$ & 0.22 & $0.11,0.35]$ & 0.17 & $0.08,0.28$ \\
\hline $\bar{l}$ & 0.23 & $-0.91,1.42]$ & 0.08 & $-1.11,1.32]$ & 0.14 & $-1.05,1.40]$ & 0.68 & $-1.13,2.53$ \\
\hline$\rho_{a}$ & 0.94 & $0.89,0.99]$ & 0.94 & $0.90,0.98]$ & 0.94 & $0.89,0.99]$ & 0.95 & $0.92,0.97$ \\
\hline$\rho_{b}$ & 0.69 & $0.55,0.82]$ & 0.77 & $0.65,0.87]$ & 0.77 & $0.65,0.87]$ & 0.23 & {$[0.09,0.38]$} \\
\hline$\rho_{g}$ & 0.48 & $0.15,0.80]$ & 0.45 & $0.13,0.78]$ & 0.47 & $0.14,0.80]$ & 0.98 & {$[0.96,0.99]$} \\
\hline$\rho_{i}$ & 0.74 & $0.51,0.97]$ & 0.77 & $0.47,0.98]$ & 0.74 & $0.36,0.98]$ & 0.72 & {$[0.62,0.82]$} \\
\hline$\rho_{r}$ & 0.19 & $0.04,0.37]$ & 0.55 & $0.21,0.91]$ & 0.34 & $0.10,0.90]$ & 0.16 & $0.05,0.27$ \\
\hline$\rho_{p}$ & 0.37 & $0.11,0.65]$ & 0.35 & $0.09,0.62]$ & 0.39 & $0.11,0.69]$ & 0.87 & $0.79,0.95$ \\
\hline$\rho_{w}$ & 0.98 & $0.95,1.00]$ & 0.97 & $0.94,1.00]$ & 0.97 & $0.94,1.00]$ & 0.96 & {$[0.93,0.98$} \\
\hline$\mu_{p}$ & 0.60 & $0.30,0.88]$ & 0.62 & $0.32,0.89]$ & 0.58 & $0.26,0.88]$ & 0.69 & {$[0.51,0.84]$} \\
\hline$\mu_{w}$ & 0.66 & $0.32,0.92]$ & 0.83 & $0.69,0.95]$ & 0.74 & {$[0.51,0.92]$} & 0.82 & {$[0.71,0.92]$} \\
\hline$\sigma_{a}$ & 0.35 & $0.31,0.41]$ & 0.34 & $0.29,0.39]$ & 0.35 & $0.31,0.40]$ & 0.44 & $0.40,0.49]$ \\
\hline$\sigma_{b}$ & 0.11 & $0.08,0.15]$ & 0.10 & $0.07,0.13]$ & 0.10 & $0.07,0.14]$ & 0.24 & {$[0.20,0.28]$} \\
\hline$\sigma_{g}$ & 0.02 & $-0.20,0.22]$ & 0.14 & $0.07,0.22]$ & 0.09 & $0.04,0.19]$ & 0.58 & {$[0.53,0.63]$} \\
\hline$\sigma_{i}$ & 0.22 & $0.09,0.34]$ & 0.17 & $0.08,0.30]$ & 0.11 & $0.04,0.27]$ & 0.41 & {$[0.34,0.50]$} \\
\hline$\sigma_{r}$ & 0.21 & $0.10,0.25]$ & 0.12 & $0.08,0.19]$ & 0.21 & $0.14,0.24]$ & 0.24 & $0.22,0.27]$ \\
\hline$\sigma_{p}$ & 0.00 & $-0.17,0.16]$ & 0.10 & $0.06,0.15]$ & 0.11 & $0.04,0.18]$ & 0.13 & $0.10,0.15]$ \\
\hline$\sigma_{w}$ & 0.02 & $-0.07,0.09]$ & 0.08 & $0.05,0.12]$ & 0.05 & $0.03,0.11]$ & 0.26 & $0.22,0.30$ \\
\hline
\end{tabular}


Table 4: Posterior median and $90 \%$ confidence bands of the three specifications, IG, IGIG and NN under different prior locations.

\begin{tabular}{|c|c|c|c|c|c|c|c|c|}
\hline & & $N N$ & & $I G I G_{*}$ & & $I G I G$ & & $I G$ \\
\hline$\iota_{w}$ & 0.55 & $0.31,0.77]$ & 0.62 & {$[0.39,0.83]$} & 0.58 & {$[0.35,0.81]$} & 0.62 & {$[0.41,0.82]$} \\
\hline$\iota_{p}$ & 0.46 & $0.23,0.71]$ & 0.49 & $0.26,0.72]$ & 0.46 & $0.22,0.69]$ & 0.30 & $0.13,0.48]$ \\
\hline$\psi$ & 0.52 & $0.33,0.72]$ & 0.51 & $0.31,0.71]$ & 0.52 & $0.32,0.72]$ & 0.61 & $0.44,0.77]$ \\
\hline$\phi_{p}$ & 1.43 & $1.30,1.57]$ & 1.42 & $1.29,1.56]$ & 1.41 & $1.28,1.55]$ & 1.71 & $1.58,1.84]$ \\
\hline$\alpha$ & 0.22 & $0.18,0.27]$ & 0.23 & $0.18,0.27]$ & 0.23 & $0.19,0.27]$ & 0.20 & $0.16,0.23]$ \\
\hline$\phi$ & 2.85 & $2.00,4.02]$ & 2.23 & $1.18,3.50]$ & 2.24 & $1.14,3.57]$ & 6.07 & $4.35,7.84]$ \\
\hline$\sigma_{c}$ & 1.03 & $0.87,1.23]$ & 1.05 & $0.88,1.25]$ & 1.03 & $0.89,1.19]$ & 1.49 & $1.24,1.75]$ \\
\hline$\sigma_{l}$ & 1.48 & $0.38,2.50]$ & 1.46 & $0.33,2.48]$ & 1.35 & $0.25,2.30]$ & 1.75 & $0.84,2.71]$ \\
\hline$\xi_{w}$ & 0.79 & $0.70,0.87]$ & 0.78 & $0.68,0.86]$ & 0.78 & $0.69,0.87]$ & 0.69 & $0.57,0.80]$ \\
\hline$\xi_{p}$ & 0.61 & $0.52,0.69]$ & 0.60 & $0.52,0.68]$ & 0.61 & $0.52,0.69]$ & 0.67 & {$[0.58,0.75]$} \\
\hline$\lambda$ & 0.76 & $0.64,0.86]$ & 0.69 & $0.55,0.83]$ & 0.73 & $0.60,0.86]$ & 0.69 & $0.62,0.76]$ \\
\hline$r_{\pi}$ & 1.97 & $1.69,2.27]$ & 2.05 & $1.78,2.35]$ & 2.03 & $1.74,2.33]$ & 2.02 & $1.73,2.32]$ \\
\hline$r_{R}$ & 0.83 & $0.78,0.88]$ & 0.82 & $0.72,0.88]$ & 0.83 & $0.74,0.89]$ & 0.81 & $0.76,0.85]$ \\
\hline$r_{y}$ & 0.15 & $0.09,0.20]$ & 0.16 & $0.11,0.22]$ & 0.16 & $0.10,0.21]$ & 0.09 & $0.05,0.12]$ \\
\hline$r_{\Delta y}$ & 0.25 & $0.20,0.29]$ & 0.24 & $0.19,0.29]$ & 0.23 & $0.18,0.28]$ & 0.22 & $0.18,0.27$ \\
\hline $\bar{\gamma}$ & 0.45 & $0.40,0.49]$ & 0.44 & $0.40,0.49]$ & 0.45 & $0.40,0.49]$ & 0.43 & {$[0.40,0.45]$} \\
\hline $\bar{\pi}$ & 0.67 & $0.51,0.84]$ & 0.68 & 0.51, & 0.67 & 0.51, & 0.83 & $0.66,1.01]$ \\
\hline $\bar{\beta}$ & 0.22 & $0.11,0.34]$ & 0.22 & $0.10,0.34]$ & 0.22 & $0.11,0.35]$ & 0.17 & $0.08,0.28$ \\
\hline $\bar{l}$ & 0.23 & $-0.91,1.42]$ & 0.08 & $-1.11,1.32]$ & 0.14 & $-1.05,1.40]$ & 0.68 & $-1.13,2.53$ \\
\hline$\rho_{a}$ & 0.94 & $0.89,0.99]$ & 0.94 & $0.90,0.98]$ & 0.94 & $0.89,0.99]$ & 0.95 & $0.92,0.97$ \\
\hline$\rho_{b}$ & 0.69 & $0.55,0.82]$ & 0.77 & $0.65,0.87]$ & 0.77 & $0.65,0.87]$ & 0.23 & {$[0.09,0.38]$} \\
\hline$\rho_{g}$ & 0.48 & $0.15,0.80]$ & 0.45 & $0.13,0.78]$ & 0.47 & $0.14,0.80]$ & 0.98 & {$[0.96,0.99]$} \\
\hline$\rho_{i}$ & 0.74 & $0.51,0.97]$ & 0.77 & $0.47,0.98]$ & 0.74 & $0.36,0.98]$ & 0.72 & {$[0.62,0.82]$} \\
\hline$\rho_{r}$ & 0.19 & $0.04,0.37]$ & 0.55 & $0.21,0.91]$ & 0.34 & $0.10,0.90]$ & 0.16 & $0.05,0.27$ \\
\hline$\rho_{p}$ & 0.37 & $0.11,0.65]$ & 0.35 & $0.09,0.62]$ & 0.39 & $0.11,0.69]$ & 0.87 & $0.79,0.95$ \\
\hline$\rho_{w}$ & 0.98 & $0.95,1.00]$ & 0.97 & $0.94,1.00]$ & 0.97 & $0.94,1.00]$ & 0.96 & {$[0.93,0.98$} \\
\hline$\mu_{p}$ & 0.60 & $0.30,0.88]$ & 0.62 & $0.32,0.89]$ & 0.58 & $0.26,0.88]$ & 0.69 & {$[0.51,0.84]$} \\
\hline$\mu_{w}$ & 0.66 & $0.32,0.92]$ & 0.83 & $0.69,0.95]$ & 0.74 & {$[0.51,0.92]$} & 0.82 & {$[0.71,0.92]$} \\
\hline$\sigma_{a}$ & 0.35 & $0.31,0.41]$ & 0.34 & $0.29,0.39]$ & 0.35 & $0.31,0.40]$ & 0.44 & $0.40,0.49]$ \\
\hline$\sigma_{b}$ & 0.11 & $0.08,0.15]$ & 0.10 & $0.07,0.13]$ & 0.10 & $0.07,0.14]$ & 0.24 & {$[0.20,0.28]$} \\
\hline$\sigma_{g}$ & 0.02 & $-0.20,0.22]$ & 0.14 & $0.07,0.22]$ & 0.09 & $0.04,0.19]$ & 0.58 & {$[0.53,0.63]$} \\
\hline$\sigma_{i}$ & 0.22 & $0.09,0.34]$ & 0.17 & $0.08,0.30]$ & 0.11 & $0.04,0.27]$ & 0.41 & {$[0.34,0.50]$} \\
\hline$\sigma_{r}$ & 0.21 & $0.10,0.25]$ & 0.12 & $0.08,0.19]$ & 0.21 & $0.14,0.24]$ & 0.24 & $0.22,0.27]$ \\
\hline$\sigma_{p}$ & 0.00 & $-0.17,0.16]$ & 0.10 & $0.06,0.15]$ & 0.11 & $0.04,0.18]$ & 0.13 & $0.10,0.15]$ \\
\hline$\sigma_{w}$ & 0.02 & $-0.07,0.09]$ & 0.08 & $0.05,0.12]$ & 0.05 & $0.03,0.11]$ & 0.26 & $0.22,0.30$ \\
\hline
\end{tabular}

